\title{
THE ASYMPTOTIC BEHAVIOR OF THE SOLUTIONS OF LINEAR AND NONLINEAR DIFFERENTIAL- DIFFERENCE EQUATIONS
}

BY

\author{
K. L. $\operatorname{COOKE}(1)$
}

1. Introduction. We intend in this paper to discuss the solutions of linear functional equations of the form

$$
\frac{d}{d t} u(t+1)=a(t) u(t)+b(t) u(t+1)
$$

and nonlinear equations of the form

$$
\frac{d}{d t} u(t+1)=a(t) u(t)+b(t) u(t+1)+D(u(t), u(t+1)),
$$

where

$$
D(u(t), u(t+1))=\sum_{i+j \geqq 2} b_{i j}(t) u(t)^{i} u(t+1)^{i} \quad(i \geqq 0, j \geqq 0),
$$

where $a(t), b(t)$, and the $b_{i j}(t)$ are given real functions of the real variable $t$. We seek to find real functions $u(t)$ which satisfy (1.1) or (1.2) for $t>t_{0}\left(t_{0}>0\right)$, and which satisfy the boundary condition $u(t)=g(t)$ for $t_{0} \leqq t \leqq t_{0}+1$, where $g(t)$ is a given real continuous function. The principal aim of this paper is to characterize such solutions for large positive values of the independent variable.

Consider, for the moment, the more general linear equation

$$
\frac{d^{n}}{d t^{n}} u(t)+\sum_{k=0}^{n-1} \sum_{i=1}^{n} c_{i k}(t) \frac{d^{k}}{d t^{k}} u\left(t-t_{i}\right)=0,
$$

where $0<t_{1}<t_{2}<\cdots<t_{n}$. As observed by Bellman [2], this equation is a special case of the system which, in vector-matrix notation, is

$$
\frac{d}{d t} u(t)=A_{0}(t) u(t)+A_{1}(t) u\left(t-t_{1}\right)+\cdots+A_{n}(t) u\left(t-t_{n}\right),
$$

where $u$ is an $n$-dimensional column vector and $A_{k}$ is a square matrix with $n$

Presented to the Society, June 21, 1952; received by the editors August 19, 1952.

(1) This investigation constituted part of the author's dissertation submitted to the Department of Mathematics and the Committee on Graduate Study of Stanford University in partial fulfillment of the requirements for the degree of Doctor of Philosophy. The author wishes to thank Professor Richard Bellman for the many valuable suggestions he has made. 
columns. The boundary condition is $u=g(t)$ for $0 \leqq t \leqq t_{n}$, where $g(t)$ is a given vector function. The methods to be employed below could be extended to provide information concerning this general equation and an associated nonlinear equation. However, in order to avoid extraneous complications, the discussion will be restricted to the first order equations (1.1) and (1.2).

Functional equations of the above type occur in several branches of mathematical physics. For references, see Hoheisel [8], Hilb [7], and Wright [17]. Very complete information on the solutions of equations with constant coefficients has been obtained by Schmidt [11], Hilb [7], Titchmarsh [12], Pitt [10], Wright $[14 ; 15 ; 17 ; 18]$, and Brownell [4], but much less is known if the coefficients are not constant. However, Bellman [2] and Wright [16] have obtained important results concerning differential-difference equations in which the coefficients, while not constants, have constant limits as $t$ tends to $+\infty$. In this paper, we suppose that $a(t)$ and $b(t)$ have asymptotic expansions

$$
a(t) \sim a_{0}+\frac{a_{1}}{t}+\frac{a_{2}}{t^{2}}+\cdots, \quad b(t) \sim b_{0}+\frac{b_{1}}{t}+\frac{b_{2}}{t^{2}}+\cdots,
$$

as $t \rightarrow+\infty$, and we seek to determine the behavior of the solutions of (1.1) and (1.2) as $t \rightarrow+\infty$. This subject is suggested by the discussion of the analogous problem for differential equations and for difference equations carried out by various authors. See, for example, Hukuhara [9] and Trjitzinsky [13], where references to related papers are given.

Before beginning the detailed study of (1.1) and (1.2), we shall briefly indicate the method to be used and the conclusions reached. Throughout this article, the equation

$$
s e^{s}-b_{0} e^{s}-a_{0}=0
$$

is called the characteristic equation, and its roots are called the characteristic roots. The first step in studying (1.1) or (1.2), where $a(t)$ and $b(t)$ have the asymptotic expansions (1.4), is to investigate the equation of first approximation

$$
\frac{d}{d t} u(t+1)=\left(a_{0}+\frac{a_{1}}{t}\right) u(t)+\left(b_{0}+\frac{b_{1}}{t}\right) u(t+1),
$$

subject to the prescribed boundary condition. By use of the Laplace transform, we may express the solution of (1.6) as a contour integral. The asymptotic behavior of the solution can then be determined by a familiar method. The principal conclusions may be stated as follows.

THeOREM 1.1. There is a unique function $u(t)$ which satisfies the equation (1.6) above for $t>t_{0}$ and which satisfies the boundary condition

$$
u(t)=g(t) \quad\left(t_{0} \leqq t \leqq t_{0}+1\right),
$$


where $g(t)$ is an arbitrary real continuous function and $a_{0}, a_{1}, b_{0}$, and $b_{1}$ are any real constants. If $a_{0} \neq-\exp \left(b_{0}-1\right)$, there is one particular characteristic root $S_{2}$, which depends on $a_{0}, a_{1}, b_{0}, b_{1}$, and $g(t)$, and there are constants $c_{0}, c_{1}, c_{2}, \cdots$, $c_{0} \neq 0$, such that, given any positive integer $m$,

$$
u(t+1)=\operatorname{Re}\left\{t^{\delta_{2}} e^{S_{2} t}\left(c_{0}+\frac{c_{1}}{t}+\cdots+\frac{c_{m}}{t^{m}}\right)\right\}+O\left(t^{\mathrm{Re}\left(\delta_{2}\right)-m-1} e^{\mathrm{Re}\left(S_{2}\right) t}\right)
$$

as $t \rightarrow+\infty$, where $\delta_{2}$ is the residue of

$$
\frac{a_{1} e^{-s}+b_{1}}{s-b_{0}-a_{0} e^{-s}}
$$

at $S_{2}$.

Theorem 1.1 is basic in the discussion of the more general equations (1.1) and (1.2), and is, perhaps, of some intrinsic interest. $\$ 2$ of this essay is devoted to the proof of Theorem 1.1. In \$3, we consider the nonhomogeneous equation

$$
\frac{d}{d t} u(t+1)=\left(a_{0}+\frac{a_{1}}{t}\right) u(t)+\left(b_{0}+\frac{b_{1}}{t}\right) u(t+1)+w(t) .
$$

By applying the Laplace transform again, we are able to prove that, under certain conditions on $w(t)$, the solution $u(t)$ of (1.10) which satisfies the boundary condition (1.7) is of the form

$$
u(t)=u_{0}(t)+u_{1}(t),
$$

where $u_{0}(t)$ is the solution of (1.6) and (1.7), and where $u_{1}(t)$ may be written in the form

$$
u_{1}(t+1)=\int_{t_{0}}^{t} w\left(t_{1}\right) k\left(t, t_{1}\right) d t_{1} .
$$

$k\left(t, t_{1}\right)$ is defined below.

In $\$ 4$, we discuss the general linear equation (1.1), where $a(t)$ and $b(t)$ have the asymptotic expansions (1.4). We note that (1.1) is of the same form as (1.10), if $w(t)$ is appropriately chosen. The results of $\$ 3$ therefore suggest the consideration of the linear integral equation

$$
u(t+1)=u_{0}(t+1)+\int_{t_{0}}^{t}\left\{A\left(t_{1}\right) u\left(t_{1}\right)+B\left(t_{1}\right) u\left(t_{1}+1\right)\right\} k\left(t, t_{1}\right) d t_{1},
$$

where $A(t)=a(t)-a_{0}-a_{1} / t$ and $B(t)=b(t)-b_{0}-b_{1} / t$. The solution of (1.11) and (1.7) may readily be constructed by the method of successive approximations, and may be shown to be the solution of (1.1). We can then ascertain its asymptotic character with the aid of Theorem 1.1 and the known form of $k\left(t, t_{1}\right)$. 
We use a similar procedure in $\$ 5$ in studying (1.2). Our principal results are as follows:

THEOREM 1.2. There is a unique function $u(t)$ which satisfies the boundary condition (1.7) and which satisfies the equation (1.1) for $t>t_{0}$, if $a(t)$ and $b(t)$ have the asymptotic expansions (1.4) as $t \rightarrow+\infty$. Let $S_{1}$ be the characteristic root of largest real part $\left({ }^{2}\right)$, and let $\delta_{1}$ be the residue of (1.9) at $S_{1}$. If $a_{0} \neq-\exp \left(b_{0}-1\right)$,

$$
u(t)=O\left(t^{\mathrm{Re}\left(\delta_{1}\right)} e^{\mathrm{Re}\left(S_{1}\right) t}\right)
$$

as $t \rightarrow+\infty\left(^{3}\right)$.

Corollary. If $\operatorname{Re}\left(S_{1}\right)<0\left({ }^{4}\right)$, or if $\operatorname{Re}\left(S_{1}\right)=0$ and $\operatorname{Re}\left(\delta_{1}\right)<0$, all solutions of (1.1) approach zero as $t \rightarrow+\infty$.

THEOREM 1.3. Consider the nonlinear equation (1.2) where $a(t)$ and $b(t)$ have the asymptotic expansions (1.4) as $t \rightarrow+\infty$, and where (1.3) holds for certain real functions $b_{i j}(t)$. Suppose that the following conditions are satisfied:

(a) $\operatorname{Re}\left(S_{1}\right)<0$;

(b) $\left|b_{i j}(t)\right| \leqq b_{i j}$, where the $b_{i j}$ are independent of $t$;

(c) $\sum_{i+j \geqq 2} b_{i j} z_{1}^{i} z_{2}^{\prime}$ converges for $\left|z_{1}\right|$ and $\left|z_{2}\right|$ sufficiently small.

Then there is a constant $C$, depending on the particular equation (1.2) under consideration, such that if

$$
\max _{t_{0} \leqq t \leqq t_{0}+1}|g(t)| \leqq C,
$$

then the following conclusions are valid: There is a unique function $u(t)$ which satisfies (1.2) for $t>t_{0}$ and which satisfies the boundary condition (1.7). $u(t)$ has the form (1.12) as $t \rightarrow+\infty\left(^{5}\right)$.

2. Proof of Theorem 1.1. Consider the equation

$$
\frac{d}{d t} u(t+1)=\left(a_{0}+\frac{a_{1}}{t}\right) u(t)+\left(b_{0}+\frac{b_{1}}{t}\right) u(t+1),
$$

in which $a_{0}, a_{1}, b_{0}, b_{1}$, and $t$ are all real. We shall, in this section, indicate the method by which Theorem 1.1 may be proved. Since the proof is obtained by standard techniques, we shall omit some of the details. In the first place, it is clear that there is a unique real function $u(t)$ which satisfies (2.1) for $t>t_{0}$ and which satisfies the boundary condition

$$
u(t)=g(t)
$$

$$
\left(t_{0} \leqq t \leqq t_{0}+1\right)
$$

(2) The existence of $S_{1}$ is proved below.

(8) The methods herein given can be extended so as to yield an asymptotic series representation.

(4) Hayes [6] has derived a necessary and sufficient condition in order that all characteristic roots have negative real parts.

(5) The methods herein given can be extended so as to yield an asymptotic series representation. 
where $g(t)$ is a given real continuous function. We shall find the Laplace transform of this solution, and then apply a standard inversion theorem in order to obtain a suitable representation of the solution. An examination of the singularities of the transform will yield the asymptotic results stated in Theorem 1.1.

First of all, it is easily shown by integrating (2.1) and then using a lemma employed extensively by other authors -cf. Bellman [1] - that

$$
|u(t+1)| \leqq C t^{\left|a_{1}\right|+\left|b_{1}\right|} e^{\left(\left|a_{0}\right|+\left|b_{0}\right|\right) t},
$$

and hence that the transform

$$
U\left(s, t_{0}\right)=\int_{t_{0}}^{\infty} u(t) e^{-s t} d t
$$

exists and is an analytic function of $s$ in a certain half-plane. From (2.1), we find that $U$ satisfies the differential equation

$$
\begin{aligned}
& \left(s e^{s}-b_{0} e^{s}-a_{0}\right) U^{\prime}\left(s, t_{0}\right) \\
& +\left[\frac{d}{d s}\left(s e^{s}-b_{0} e^{s}-a_{0}\right)+a_{1}+b_{1} e^{s}\right] U\left(s, t_{0}\right)=G\left(s, t_{0}\right)
\end{aligned}
$$

within the half-plane of convergence, where

$$
\begin{aligned}
G\left(s, t_{0}\right)= & -t_{0} g\left(t_{0}+1\right) e^{-s t_{0}}-\left(s-b_{0}\right) e^{s} \int_{t_{0}}^{t_{0}+1} t g(t) e^{-s t} d t \\
& +e^{s}\left(s+1-b_{0}+b_{1}\right) \int_{t_{0}}^{t_{0}+1} g(t) e^{-s t} d t .
\end{aligned}
$$

Before solving (2.4), we must make a few preliminary remarks, the first of which concern the characteristic roots, the roots of

$$
s e^{s}-b_{0} e^{s}-a_{0}=0 \text {. }
$$

Wright [14] proved that in any vertical strip $x_{1} \leqq \operatorname{Re}(s) \leqq x_{2}$, there are but a finite number of characteristic roots. It is also easily established that if $a_{0} \neq-\exp \left(b_{0}-1\right)$, all the characteristic roots are simple, and that on any vertical line $\operatorname{Re}(s)=x$, either there is a single root, and it is $x$, or there are two conjugate complex roots, or there are no roots. Finally, all the roots $S$ are such that $\operatorname{Re}(S) \leqq\left|a_{0}\right|+\left|b_{0}\right|$. Thus there is a characteristic root $S_{1}=X_{1}+i Y_{1}$ with the property that all the characteristic roots lie on the line $\operatorname{Re}(s)=X_{1}$, or to the left of it.

Let $x_{0}$ be an arbitrary real number for which $x_{0}>X_{1}$, and let $s$ be any complex number which is not a characteristic root. Define the function

$$
h(s)=\int_{x_{0}, L_{1}}^{s} \frac{a_{1} e^{-s_{1}}+b_{1}}{s_{1}-b_{0}-a_{0} e^{-s_{1}}} d s_{1}
$$


where $L_{1}$ is a contour of the following description. $L_{1}$ starts at $x_{0}$ and ends at $s$, and $\operatorname{Re}\left(s_{1}\right) \geqq \operatorname{Re}(s)$ all along $L_{1} . L_{1}$ does not pass through any characteristic root. Furthermore, if $\operatorname{Im}(s) \geqq 0, L_{1}$ passes above all characteristic roots $s^{\prime}$ for which $\operatorname{Re}\left(s^{\prime}\right)>\operatorname{Re}(s)$. If $\operatorname{Im}(s)<0, L_{1}$ passes below all characteristic roots $s^{\prime}$ for which $\operatorname{Re}\left(s^{\prime}\right)>\operatorname{Re}(s)$. The function $h(s)$ is thus defined and single-valued except at the characteristic roots. It is not difficult to see that $h(s)$ will, in general, have branch points at the characteristic roots. If $s^{\prime}$ is any characteristic root and if $\delta^{\prime}$ is the residue of $h^{\prime}(s)$ at $s^{\prime}$,

$$
\exp \{h(s)\}=\left(s-s^{\prime}\right)^{\delta^{\prime}} \sum_{n=0}^{\infty} d_{n}\left(s-s^{\prime}\right)^{n}
$$

for $s$ "near $s^{\prime}$." If $\delta^{\prime}$ is a non-negative integer, (2.8) is valid in a circle $\left|s-s^{\prime}\right|$ $\leqq C$, but if $\delta^{\prime}$ is a negative integer an expansion of the above form holds only in an annulus $0<\left|s-s^{\prime}\right| \leqq C$. If $\delta^{\prime}$ is not an integer, exp $\{h(s)\}$ has a branch point at $s^{\prime}$, and (2.8) is valid in an annulus $0<\left|s-s^{\prime}\right| \leqq C$, cut along the real axis if $s^{\prime}$ is real, but along the line joining $s^{\prime}$ and $\bar{s}^{\prime}$ if $s^{\prime}$ is not real. Also, the expansion

$$
\frac{e^{-h(s)}}{s-b_{0}-a_{0} e^{-s}}=\left(s-s^{\prime}\right)^{-\delta^{\prime}-1} \sum_{n=0}^{\infty} h_{n}\left(s-s^{\prime}\right)^{n}
$$

is valid in an appropriate neighborhood of $s^{\prime}$.

The following inequalities, which may be proved without great difficulty, will be needed below. First, given any $\sigma>X_{1}$, there are constants $C_{1}, C_{2}$, and $p$ such that

$$
\left|s-b_{0}-a_{0} e^{-s}\right| \geqq C_{1}|s|,|\exp h(s)| \leqq C_{2}|s|^{p},
$$

and

$$
|\exp \{-h(s)\}| \leqq C_{2}|s|^{p}
$$

for $\operatorname{Re}(s) \geqq \sigma$. Given any real $\sigma$, there are real numbers $\rho$ and $C$, depending on $\sigma$, for which

$$
\left|\exp \left\{h\left(x_{2}+i \omega\right)-h\left(x_{1}+i \omega\right)\right\}\right| \leqq \exp \left\{\left(C+C e^{-x_{1}}\right)\left(x_{2}-x_{1}\right)\right\}
$$

for all $x_{1}, x_{2}$, and $\omega$ such that $x_{2}>x_{1} \geqq \sigma$ and $|\omega|>\rho$. Finally, from (2.5) we find that

$$
\left|G\left(s, t_{0}\right)\right| \leqq C e^{x\left(1-t_{0}\right)},
$$

for $x=\operatorname{Re}(s) \geqq \sigma$.

The solution of (2.4) which we require is one which approaches zero as $s$ tends to infinity along the positive real axis. The only such solution is

$$
U\left(s, t_{0}\right)=\frac{e^{-h(s)}}{s e^{s}-b_{0} e^{s}-a_{0}} \int_{+\infty+i 0, L_{2}}^{s} G\left(s_{1}, t_{0}\right) e^{h\left(s_{1}\right)} d s_{1},
$$


where $L_{2}$ denotes any contour from $+\infty+i 0$ to $s=x+i y$ which lies on or to the right of the line of abscissa $x$. In order to show that this solution does approach zero as $s$ tends to infinity along the real axis, we use the above inequalities. It is necessary to suppose that $t_{0}$ is sufficiently large. This is no loss of generality, since we may successively compute the solution in the intervals $\left(t_{0}, t_{0}+1\right), \cdots,\left(t_{0}+m, t_{0}+m+1\right)$, using (2.1) and (2.2), and take as a new initial condition the functional values found for the interval $\left(t_{0}+m, t_{0}+m+1\right)$.

From (2.13) we can immediately obtain a representation of the solution $u(t)$ of $(2.1)$, using the inversion theorem stated in Doetsch [5, p. 107]. It is necessary to prove only that, if $L$ is the vertical line from $x-i \infty$ to $x+i \infty$,

$$
\int_{L} e^{t_{s}} U\left(s, t_{0}\right) d s
$$

is uniformly convergent in the interval $T \leqq t \leqq T_{2}$ for any $T>t_{0}+1$ and any finite $T_{2}>T$, provided $x$ is sufficiently large. This may be accomplished by integrating by parts and using the inequalities given above. Thus we find that

$$
u(t)=\frac{1}{2 \pi i} \int_{L} e^{t_{0}} U\left(s, t_{0}\right) d s \quad\left(t>t_{0}+1\right) .
$$

The formula (2.13) may be used to continue $U\left(s, t_{0}\right)$ analytically into the whole $s$-plane, cut along the lines of discontinuity of $\exp h(s)$, if we require $L_{2}$ to be a contour of the same type as $L_{1}$. Let $s^{\prime}$ be any characteristic root, let $\delta^{\prime}$ be the residue of $h^{\prime}(s)$ at $s^{\prime}$, and suppose that $\delta^{\prime}$ is not a negative integer. Then by integrating by parts $n$ times and using (2.8) and (2.9), we find from (2.13) that

$$
\begin{aligned}
U\left(s, t_{0}\right)= & \frac{e^{-s} e^{-h(s)}}{s-b_{0}-a_{0} e^{-s}} \sum_{j=0}^{n-1}(-1)^{j} G^{(j)}\left(s, t_{0}\right) H_{j+1}(s) \\
& +\frac{(-1)^{n} e^{-s} e^{-h(s)}}{s-b_{0}-a_{0} e^{-s}} \int_{s^{\prime}}^{0} G^{(n)}\left(s_{1}, t_{0}\right) H_{n}\left(s_{1}\right) d s_{1} \\
& +J_{n} e^{-s}\left(s-s^{\prime}\right)^{-\delta^{\prime}-1} \sum_{n=0}^{\infty} h_{n}\left(s-s^{\prime}\right)^{n} .
\end{aligned}
$$

This equation is valid in an annulus $0<\left|s-s^{\prime}\right| \leqq C$, or in an annulus cut along a certain line segment, according to the value of $\delta^{\prime}$. In this formula $H_{0}(s)=\exp h(s)$ and $H_{n+1}(s)$ is an indefinite integral of $H_{n}(s)$ for $n$ $=0,1,2, \cdots$ Also, each $H_{j}(s)$ has an expansion

$$
H_{j}(s)=\left(s-s^{\prime}\right)^{\delta^{\prime}+i} \sum_{k=0}^{\infty} \frac{d_{k}\left(s-s^{\prime}\right)^{k}}{\left(\delta^{\prime}+k+1\right) \cdots\left(\delta^{\prime}+k+j\right)},
$$


valid in an appropriate neighborhood of $s^{\prime} . J_{n}$ is defined by

$$
J_{n}=J_{n}\left(s^{\prime}, t_{0}\right)=(-1)^{n} \int_{+\infty+i 0}^{s^{\prime}} G^{(n)}\left(s_{1}, t_{0}\right) H_{n}\left(s_{1}\right) d s_{1},
$$

where the path is of the same type as $L_{2}$. This integral converges if $n+\operatorname{Re}\left(\delta^{\prime}\right)$ $>-1$, according to (2.16), as does the integral in (2.15).

A formula similar to (2.15) holds for $s$ near $\bar{s}^{\prime} . s^{\prime}, J_{n}, \delta^{\prime}$, and the $h_{j}$ must be replaced by their conjugates, and the functions $H_{j}(s)$ must be replaced by functions $H_{j}^{*}(s)$ with expansions

$$
H_{j}^{*}(s)=\left(s-\bar{s}^{\prime}\right)^{\bar{\delta}^{\prime}+j} \sum_{k=0}^{\infty} \frac{\bar{d}_{k}\left(s-\bar{s}^{\prime}\right)^{k}}{\left(\bar{\delta}^{\prime}+k+1\right) \cdots\left(\bar{\delta}^{\prime}+k+j\right)}
$$

near $\bar{s}^{\prime}$.

We now let $S_{2}=X_{2}+i Y_{2}$ denote the characteristic root of largest real part at which $U$ has a singularity. We shall define certain functions whose transforms comprise the "principal part" of $U$ at $S_{2}$ and $\bar{S}_{2}$. Let $\delta_{2}$ be the residue of $h^{\prime}(s)$ at $S_{2}$. We shall first suppose that $\delta_{2}$ is not an integer, and shall consider two cases.

Case 1. Re $\left(\delta_{2}\right)>-1, \delta_{2}$ not an integer. Let any positive integer $n$ be chosen such that $n+\operatorname{Re}\left(\delta_{2}\right)>1$. Let $\nu$ denote the greatest integer less than $\operatorname{Re}\left(\delta_{2}\right)$. Define

$$
\begin{array}{rlrl}
r_{j}(t)=\frac{t^{\delta_{2}-j} e^{S_{2} t}}{\Gamma\left(\delta_{2}+1-j\right)} & (t \geqq 0 ; j=0,1, \cdots, \nu+1), \\
r_{j}(t)=\left\{\begin{array}{cl}
0 & (0 \leqq t<1) \\
\frac{t^{\delta_{2}-j_{e} S_{2} t}}{\Gamma\left(\delta_{2}+1-j\right)} & (t \geqq 1)
\end{array} \quad(j=\nu+2, \cdots, \nu+n+2) .\right.
\end{array}
$$

All the functions $r_{j}(t)$ are defined as zero for $-1 \leqq t<0$. Define

$$
r(t)=\sum_{j=0}^{v+n+2} h_{j} r_{j}(t)
$$

and define $R(s)$ to be the transform of $r(t)$. The transform will converge for $\operatorname{Re}(s)>X_{2}$, and it is well known that

$$
R(s)=\left(s-S_{2}\right)^{-\delta_{2}-1} \sum_{j=0}^{n+n+2} h_{j}\left(s-S_{2}\right)^{i}+\text { entire function. }
$$

This equation provides a continuation of $R(s)$ into the whole $s$-plane, cut along certain lines. Also, if $R_{c}(s)$ denotes the transform of $\bar{r}(t), R_{c}(s)$ is given by an expression of the form of (2.21), but with $S_{2}, \delta_{2}, h_{0}, h_{1}, \cdots$ replaced by their conjugates. Finally, define 
(2.22) $f(t-1)=f\left(t-1, t_{0}\right)=\left\{\begin{array}{cr}0 & (0 \leqq t<1), \\ -J_{n} r(t-1)-\bar{J}_{n} \bar{r}(t-1) & \left(1<t<t_{0}\right), \\ u(t)-J_{n} r(t-1)-\bar{J}_{n} \bar{r}(t-1) & \left(t_{0} \leqq t\right) .\end{array}\right.$

The Laplace transform of this function exists for sufficiently large $x$, and is

$$
\begin{aligned}
F(s) & =F\left(s, t_{0}\right)=\int_{0}^{\infty} f(t-1) e^{-s t} d t \\
& =U\left(s, t_{0}\right)-J_{n} e^{-s} R(s)-\bar{J}_{n} e^{-s} R_{c}(s) .
\end{aligned}
$$

(2.24) may be taken as the definition of $F\left(s, t_{0}\right)$ over the whole $s$-plane, cut along the lines of discontinuity of $U$.

Case 2. $\operatorname{Re}\left(\delta_{2}\right) \leqq-1, \delta_{2}$ not an integer. In this case we define $r_{j}(t)$ by (2.19) for $j=0,1, \cdots, n$, and take the sums in (2.20) and (2.21) from $j=0$ to $j=n$, but otherwise the discussion is unaltered.

We shall now apply a slightly extended form of a theorem stated in Doetsch [5, p. 269, Satz 4]. We note the following facts:

(a) $F\left(s, t_{0}\right)$ is analytic for $\operatorname{Re}(s)>X_{2}$ and for $\operatorname{Re}(s)=X_{2}, \operatorname{Im}(s)>Y_{2}$. As $\operatorname{Re}(s) \rightarrow X_{2}$ from the right, $F\left(s, t_{0}\right)$ possesses boundary values, denoted by $F\left(X_{2}+i y, t_{0}\right)$ or $F\left(X_{2}+i y\right)$, in the sense that the function $F\left(s, t_{0}\right)$ completed in the half-plane $\operatorname{Re}(s) \geqq X_{2}$ by these values is a continuous function. $F\left(X_{2}+i y\right)$ is $n$ times differentiable with respect to $y$, and the $n$th derivative is continuous for all $y$. This may be deduced from the expansions (2.15) and (2.21).

(b) The integral

$$
\int_{X_{2}+i \omega}^{x+i \omega} e^{t_{s}} U\left(s, t_{0}\right) d s
$$$$
\left(x>X_{2} ; t>t_{0}\right)
$$

approaches zero as $|\omega| \rightarrow \infty$. This follows from (2.13) and the inequalities (2.10), (2.11), and (2.12).

(c) $U\left(X_{2}+i y\right)$ and its first $n-1$ derivatives with respect to $y$ approach zero as $|y| \rightarrow \infty$. The proof of this fact is obtained by computing the derivatives of $U\left(s, t_{0}\right)$ and utilizing the following facts.

$$
\frac{d^{j}}{d s^{j}} e^{h(s)}=e^{h(s)} \phi_{j}(s)
$$

where

$$
\left|\phi_{j}\left(X_{2}+i y\right)\right| \leqq\left\{\begin{array}{cr}
\frac{C}{\left|X_{2}+i y\right|} \\
C & (j=1,2, \cdots),
\end{array}\right.
$$

and 


$$
\frac{d^{j}}{d s^{j}}\left(\frac{e^{-h(s)}}{s e^{s}-b_{0} e^{s}-a_{0}}\right)=e^{-h(s)} \psi_{j}(s)
$$

where

$$
\left|\psi_{j}\left(X_{2}+i y\right)\right| \leqq \frac{C}{\left|X_{2}+i y\right|} \quad(j=1,2, \cdots) .
$$

From (a), (b), and (c), we may deduce, as in Doetsch, that

$$
f(t-1)=\frac{i^{n}}{2 \pi} \frac{e^{X_{2} t}}{t^{n}} \int_{-\infty}^{\infty} e^{i t y}\left\{\frac{d^{n}}{d y^{n}} F\left(X_{2}+i y\right)\right\} d y
$$

for $t>t_{0}+1$. Finally, we observe that the integrals

$$
\int_{-\infty}^{-\eta} e^{i t y}\left\{\frac{d^{n}}{d y^{n}} U\left(X_{2}+i y\right)\right\} d y \text { and } \int_{\eta}^{\infty} e^{i t y}\left\{\frac{d^{n}}{d y^{n}} U\left(X_{2}+i y\right)\right\} d y,
$$

where $\eta$ is a fixed number greater than $Y_{2}$, are uniformly convergent for $t$ $\geqq T(T>0)$. This is readily proved. Consequently, we find, by arguing as in Doetsch, that

$$
\lim _{t \rightarrow 0} t^{n} e^{-X_{2} t}\left[u(t)-J_{n} r(t-1)-\bar{J}_{n} \bar{r}(t-1)\right]=0 .
$$

This result is valid for any positive integer $n$ for which $n+\operatorname{Re}\left(\delta_{2}\right)>1$, provided $\delta_{2}$ is not an integer. Using the definitions of $r(t)$, we obtain the asymptotic formula (1.8) of Theorem 1.1.

It is still necessary to consider the case in which $\delta_{2}$ is an integer. If $\delta_{2}$ is a non-negative integer, we may obtain equation (1.8) by abandoning the above method and using the residue theorem. If $\delta_{2}$ is a negative integer, equations (2.13) and (2.14) are still valid, but instead of (2.15) we have

$$
U\left(s, t_{0}\right)=J e^{-s}\left(s-S_{2}\right)^{-\delta_{2}-1} \log \left(s-S_{2}\right) \sum_{j=0}^{\infty} h_{j}\left(s-S_{2}\right)^{j}+\sum_{j=0}^{\infty} C_{j}\left(s-S_{2}\right)^{j},
$$

where $J$ is the residue of $G(s) \exp (h(s))$ at $S_{2}$. By defining

$$
r_{j}(t)=\left\{\begin{array}{cr}
0 & (-1 \leqq t<1), \\
(-1)^{q+j}(q-1) ! t^{-q} e^{S_{2} t} & (t \geqq 1)
\end{array}\right.
$$

for $j=0,1, \cdots, n+1-q$, where $q=-\delta_{2}$, and proceeding as before, we again find that equation (1.8) holds. This completes the proof of Theorem 1.1.

3. The nonhomogeneous equation. In this section, we shall consider the equation

$$
\frac{d}{d t} u(t+1)=\left(a_{0}+\frac{a_{1}}{t}\right) u(t)+\left(b_{0}+\frac{b_{1}}{t}\right) u(t+1)+w(t)
$$


where $w(t)$ is a given continuous function, subject to the boundary condition

$$
u(t)=g(t) \quad\left(t_{0} \leqq t \leqq t_{0}+1\right) .
$$

The principal results relating to equation (3.1) are stated in Theorem 3.1 at the end of this section. They form a necessary preliminary to the discussions in $\$ \S 4$ and 5 .

We first observe that there is clearly a unique solution of (3.1) and (3.2). Let us apply the Laplace transform formally to (3.1), as in \$2 of this paper, solve the resulting differential equation, and employ the customary inversion. The function

$$
u(t)=u_{0}(t)+u_{1}(t)
$$

is thus suggested as a possible solution of (3.1), where $u_{0}(t)$ is the solution of the homogeneous equation discussed in the preceding section and where

$$
\begin{aligned}
& u_{1}(t)=-\frac{1}{2 \pi i} \int_{L} \frac{e^{t_{s}} e^{-h(s)}}{s e^{s}-b_{0} e^{s}-a_{0}} \\
& \cdot\left\{\int_{+\infty+i 0, L_{2}}^{s} e^{h\left(s_{1}\right)}\left(\int_{t_{0}}^{\infty} t_{1} w\left(t_{1}\right) e^{-s_{1} t_{1}} d t_{1}\right) d s_{1}\right\} d s .
\end{aligned}
$$

Before showing that (3.3) actually gives the desired solution, we observe that, if $w(t)$ is continuous for $t>t_{0}$ and if

$$
\int_{t_{0}}^{\infty} t_{1} w\left(t_{1}\right) e^{-s t_{1}} d t_{1}
$$

is absolutely convergent for $\operatorname{Re}(s)>X_{1}$, the integrals

$$
\int_{+\infty+i 0}^{0} e^{h\left(s_{1}\right)}\left(\int_{t_{0}}^{\infty} t_{1} w\left(t_{1}\right) e^{-s_{1} t_{1}} d t_{1}\right) d s_{1}
$$

and

$$
\int_{t_{0}}^{\infty} t_{1} w\left(t_{1}\right)\left(\int_{+\infty+i 0}^{0} e^{h\left(\theta_{1}\right)} e^{-s_{1} t_{1}} d s_{1}\right) d t_{1}
$$

exist for $x=\operatorname{Re}(s)>X_{1}$, and are equal. This follows from standard theorems on interchange of order. Moreover,

$$
\int_{-\infty}^{\infty} \frac{e^{i \tau y}}{x+i y-b_{0}-a_{0} e^{-x-i y}} d y
$$

is uniformly convergent in every finite interval $\tau_{1} \leqq \tau \leqq \tau_{2}$, where $\tau_{1}>0\left[\tau_{2}<0\right]$, and boundedly convergent for $\tau>0[\tau<0]$, provided $x>X_{1}$. We can also show that 


$$
\int_{L} \frac{e^{t s} e^{-h(s)}}{s-b_{0}-a_{0} e^{-s}}\left(\int_{+\infty+i 0}^{s} e^{h\left(s_{1}\right)} e^{-s_{1} t_{1}} d s_{1}\right) d s=0,
$$

if $t_{1}>t \geqq t_{0}-1$, by integrating the inner integral by parts and shifting the contour $L$ arbitrarily far to the right. It therefore follows from standard theorems that

$-2 \pi i u_{1}(t+1)$

$$
\begin{aligned}
& =\int_{L} \frac{e^{t_{s}} e^{-h(s)}}{s-b_{0}-a_{0} e^{-s}}\left\{\int_{t_{0}}^{\infty} t_{1} w\left(t_{1}\right)\left(\int_{L_{2}} e^{h\left(s_{1}\right)} e^{-s_{1} t_{1}} d s_{1}\right) d t_{1}\right\} d s \\
& =\int_{L} \frac{e^{t 8} e^{-h(s)}}{s-b_{0}-a_{0} e^{-s}}\left\{\int_{L_{2}} e^{h\left(s_{1}\right)}\left(\int_{l_{0}}^{\infty} t_{1} w\left(t_{1}\right) e^{-s_{1} t_{1}} d t_{1}\right) d s_{1}\right\} d s \\
& =\int_{t_{0}}^{\infty} t_{1} w\left(t_{1}\right)\left\{\int_{L} \frac{e^{t_{s}} e^{-h(s)}}{s-b_{0}-a_{0} e^{-s}}\left(\int_{L_{2}} e^{h\left(s_{1}\right)} e^{-s_{1} t_{1}} d s_{1}\right) d s\right\} d t_{1} \\
& =\int_{t_{0}}^{t} t_{1} w\left(t_{1}\right)\left\{\int_{L} \frac{e^{t_{s}} e^{-h(s)}}{s-b_{0}-a_{0} e^{-s}}\left(\int_{L_{2}} e^{h\left(s_{1}\right)} e^{-s_{1} t_{1}} d s_{1}\right) d s\right\} d t_{1} \text {, }
\end{aligned}
$$

where each integral exists for $t>t_{0}$ and $x>X_{1}$. The path $L$ is the straight line from $x-i \infty$ to $x+i \infty$.

We shall now prove that the function $u(t)$ defined by (3.3) is the unique solution of (3.1) and (3.2). In order to prove that $u(t)$ satisfies the boundary condition, we must demonstrate that $u_{1}(t)=0$ for $t_{0} \leqq t \leqq t_{0}+1$. This follows, for $t_{0} \leqq t<t_{0}+1$, from the third equation of (3.6) by shifting $L$ to the right. $u_{1}\left(t_{0}+1\right)$ may be defined to be zero.

It must still be proved that $u(t+1)$ is a solution of (3.1) for $t>t_{0}$. Since $u_{0}(t)$ is a solution of the homogeneous equation discussed in $\$ 2$, it will be enough to show that $u_{1}(t)$ is a particular solution of (3.1). It seems to be difficult to do this directly. Instead, we adopt the following device. Let

$$
\begin{aligned}
I_{1}(t)= & u_{1}(t+1)-a_{0} \int_{t_{0}}^{t} u_{1}\left(t_{1}\right) d t_{1}-a_{1} \int_{t_{0}}^{t} \frac{u_{1}\left(t_{1}\right)}{t_{1}} d t_{1} \\
& -b_{0} \int_{t_{0}}^{t} u_{1}\left(t_{1}+1\right) d t_{1}-b_{1} \int_{t_{0}}^{t} \frac{u_{1}\left(t_{1}+1\right)}{t_{1}} d t_{1}-\int_{t_{0}}^{t} w\left(t_{1}\right) d t_{1} .
\end{aligned}
$$

We shall now show that $I_{1}(t)$ is identically zero for $t>t_{0}$. The required result will then follow by differentiation. For convenience, define

$$
k\left(t, t_{1}\right)=-\frac{t_{1}}{2 \pi i} \int_{L} \frac{e^{t_{s}} e^{-h(s)}}{s-b_{0}-a_{0} e^{-s}}\left(\int_{L_{2}} e^{h\left(s_{1}\right)} e^{-s_{1} t_{1}} d s_{1}\right) d s
$$

for $t$ and $t_{1}$ greater than $t_{0}-1$. As shown above, $k\left(t, t_{1}\right)$ is independent of $x$ as long as $x>X_{1}$, and $k\left(t, t_{1}\right)=0$ for $t_{1}>t \geqq t_{0}-1$. By bounded convergence of the 
integral, $k\left(t, t_{1}\right)$ is a continuous function of $t$ and $t_{1}$ as long as $t>t_{1} \geqq t_{0}-1$, and therefore $u_{1}(t)$ is continuous. From (3.6),

$$
u_{1}(t+1)=\int_{t_{0}}^{t} w\left(t_{1}\right) k\left(t, t_{1}\right) d t_{1} .
$$

Now if $q\left(t_{1}, t_{2}\right)$ is any function that is continuous in $t_{1}$ and $t_{2}$ for $t \geqq t_{1}>t_{2} \geqq t_{0}$, it is easy to see that

$$
\int_{t_{0}}^{t} d t_{1} \int_{t_{0}}^{t_{1}} q\left(t_{1}, t_{2}\right) d t_{2}=\int_{t_{0}}^{t} d t_{2} \int_{t_{2}}^{t} q\left(t_{1}, t_{2}\right) d t_{1} .
$$

By using this equation and (3.9) we can show that

$$
\begin{aligned}
\int_{t_{0}}^{t} u_{1}\left(t_{1}\right) d t_{1}=-\frac{1}{2 \pi i} \int_{L} \frac{e^{(t-1) s} e^{-h(s)}}{s\left(s-b_{0}-a_{0} e^{-s}\right)} \\
\cdot\left\{\int_{L_{2}} e^{h\left(s_{1}\right)}\left(\int_{t_{0}}^{\infty} t_{2} w\left(t_{2}\right) e^{-s_{1} t_{2}} d t_{2}\right) d s_{1}\right\} d s .
\end{aligned}
$$

Formulas for the other integrals appearing in (3.7) may be deduced directly from (3.4). Combining these results yields the equation

$$
\begin{aligned}
2 \pi i I_{1}(t)= & -\int_{L} \frac{e^{t_{s}} e^{-h(s)}}{s}\left\{\int_{L_{2}} e^{h\left(s_{1}\right)}\left(\int_{t_{0}}^{\infty} t_{2} w\left(t_{2}\right) e^{-s_{1} t_{2}} d t_{2}\right) d s_{1}\right\} d s \\
& +\int_{t_{0}}^{t} \frac{d t_{1}}{t_{1}} \int_{L} e^{t_{1} s} h^{\prime}(s) e^{-h(s)} \\
& \cdot\left\{\int_{L_{2}} e^{h\left(s_{1}\right)}\left(\int_{t}^{\infty} t_{2} w\left(t_{2}\right) e^{-s_{1} t_{2}} d t_{2}\right) d s_{1}\right\} d s \\
& -2 \pi i \int_{t_{0}}^{t} w\left(t_{1}\right) d t_{1}
\end{aligned}
$$

We now integrate the integral over $L_{2}$ by parts, use Doetsch $[5$, p. 107, Theorem 5], and change the order of integration, obtaining

$$
\begin{aligned}
2 \pi i I_{1}= & -\int_{t_{0}}^{t} w\left(t_{2}\right) d t_{2} \int_{L} \frac{e^{t_{s}} e^{-h(s)}}{s}\left(\int_{L_{2}} e^{h\left(s_{1}\right)} h^{\prime}\left(s_{1}\right) e^{-s_{1} t_{2}} d s_{1}\right) d s \\
+ & \int_{t_{0}}^{t} \frac{d t_{1}}{t_{1}} \int_{t_{0}}^{t_{1}} t_{2} w\left(t_{2}\right) d t_{2} \int_{L} e^{t_{1} \bullet} h^{\prime}(s) e^{-h(s)} \\
& \cdot\left(\int_{L_{2}} e^{h\left(s_{1}\right)} e^{-s_{1} t_{2}} d s_{1}\right) d s .
\end{aligned}
$$

We now let $I_{2}\left(t_{1}, t_{2}\right)$ denote the integral over $L$ appearing in the second term above. Using integration by parts, we find that 


$$
\begin{aligned}
I_{2} & =-\frac{1}{t_{2}} \int_{L} e^{t_{1 s} s}\left\{\frac{d}{d s}\left(e^{-h(s)} \int_{L_{2}} e^{h\left(s_{1}\right)} h^{\prime}\left(s_{1}\right) e^{-s_{1} t_{2}} d s_{1}\right)\right\} d s \\
& =\frac{t_{1}}{t_{2}} \int_{L} e^{t_{18} s} e^{-h(s)}\left(\int_{L_{2}} e^{h\left(s_{1}\right)} e^{-s_{1} t_{2}} d s_{1}\right) d s .
\end{aligned}
$$

By using this result in the second term of (3.12), then using (3.10), and finally interchanging the order of integration, we find that $I_{1}(t)=0$ for $t>t_{0}$, as we set out to prove. Therefore $u(t)$ is the solution of (3.1) and (3.2).

The results of this section are summarized in the theorem below.

THEOREM 3.1. If $w(t)$ is continuous for $t>t_{0}$ and if

$$
\int_{t_{0}}^{\infty} t_{1} w\left(t_{1}\right) e^{-s t_{1}} d t_{1}
$$

is absolutely convergent for $\operatorname{Re}(s)>X_{1}$, the unique function which satisfies (3.1) for $t>t_{0}$ and which satisfies (3.2) for $t_{0} \leqq t \leqq t_{0}+1$ is

$$
u(t)=u_{0}(t)+u_{1}(t) .
$$

Here $u_{0}(t)$ is the function discussed in $\$ 2$, and for $t>t_{0}$

$$
u_{1}(t+1)=\int_{t_{0}}^{t} w\left(t_{1}\right) k\left(t, t_{1}\right) d t_{1},
$$

where $k\left(t, t_{1}\right)$ is defined by (3.8).

4. The general linear equation. We now come to the proof of the principal results of this article as stated in Theorems 1.2 and 1.3. We shall construct a function $u(t)$ which, for $t>t_{0}$, satisfies the equation

$$
\frac{d}{d t} u(t+1)=a(t) u(t)+b(t) u(t+1)
$$

where $a(t)$ and $b(t)$ have asymptotic expansions

$$
a(t) \sim a_{0}+\frac{a_{1}}{t}+\frac{a_{2}}{t^{2}}+\cdots, \quad b(t) \sim b_{0}+\frac{b_{1}}{t}+\frac{b_{2}}{t^{2}}+\cdots,
$$

as $t \rightarrow+\infty$, and which satisfies the condition

$$
u(t)=g(t)
$$

for $t_{0} \leqq t \leqq t_{0}+1$. It is clear that such a function exists, and is unique. Since equation (4.1) may be written in the form of equation (3.1), where $w(t)$ $=A(t) u(t)+B(t) u(t+1)$ and

$$
A(t) \sim \frac{a_{2}}{t^{2}}+\frac{a_{3}}{t^{3}}+\cdots, \quad B(t) \sim \frac{b_{2}}{t^{2}}+\frac{b_{3}}{t^{3}}+\cdots,
$$


the results of the preceding section suggest the consideration of the integral equation

$$
u(t+1)=u_{0}(t+1)+\int_{t_{0}}^{t}\left\{A\left(t_{1}\right) u\left(t_{1}\right)+B\left(t_{1}\right) u\left(t_{1}+1\right)\right\} k\left(t, t_{1}\right) d t_{1} .
$$

$u_{0}(t)$ and $k\left(t, t_{1}\right)$ are defined above.

We shall construct the unique solution of $(4.5)$ by the method of successive approximations, and deduce Theorem 1.2 from the results. Before we can do this, however, we must obtain some further information concerning $k\left(t, t_{1}\right)$. This will be done by a method similar to that employed in $\$ 2$. First we observe that the Laplace transform

$$
K\left(s, t_{1}\right)=\int_{0}^{\infty} k\left(t, t_{1}\right) e^{-s t} d t=\int_{t_{1}}^{\infty} k\left(t, t_{1}\right) e^{-s t} d t
$$

exists for $\operatorname{Re}(s)>X_{1}$, and by (3.8) and an extended form of Cauchy's Integral Formula,

$$
K\left(s, t_{1}\right)=-\frac{t_{1} e^{-h(s)}}{s-b_{0}-a_{0} e^{-s}} \int_{+\infty+i 0, L_{2}}^{s} e^{h\left(s_{1}\right)} e^{-s_{1} t_{1}} d s_{1} .
$$

(4.7) provides an analytic continuation of $K\left(s, t_{1}\right)$ into the whole $s$-plane, cut along various lines. Let $s^{\prime}$ be any characteristic root, let $\delta^{\prime}$ be the residue of $h^{\prime}(s)$ at $s^{\prime}$, and define

$$
J_{n}\left(t_{1}\right)=J_{n}\left(s^{\prime}, t_{1}\right)=t_{1}^{n} \int_{+\infty+i 0}^{s^{\prime}} H_{n}\left(s_{1}\right) e^{-s_{1} t_{1}} d s_{1} .
$$

Exactly as in $\S 2$, we find that for any $s$ in a certain annulus $0<\left|s-s^{\prime}\right| \leqq C$, cut along the line of discontinuity of $K$, if any,

$$
\begin{aligned}
-\frac{K\left(s, t_{1}\right)}{t_{1}}= & \frac{e^{-h(s)} e^{-s t_{1}}}{s-b_{0}-a_{0} e^{-s}} \sum_{j=1}^{n} H_{j}(s) t_{1}^{j-1} \\
& +\frac{t_{1}^{n} e^{-h(s)}}{s-b_{0}-a_{0} e^{-s}} \int_{s^{\prime}}^{0} H_{n}\left(s_{1}\right) e^{-s_{1} t_{1}} d s_{1} \\
& +J_{n}\left(t_{1}\right)\left(s-s^{\prime}\right)^{-\delta^{\prime}-1} \sum_{j=0}^{\infty} h_{j}\left(s-s^{\prime}\right)^{i},
\end{aligned}
$$

provided $\delta^{\prime}$ is not a negative integer. In the cut annulus $0<\left|s-\bar{s}^{\prime}\right| \leqq C$, a similar formula is valid; the $H_{n}(s)$ must be replaced by related functions and $s^{\prime}, J_{n}, \delta^{\prime}$, and the $h_{j}$ must be replaced by their conjugates. The path of integration in (4.9) is the line joining $s^{\prime}$ and $s$.

As in $\S 2$, the cases in which $\delta^{\prime}$ is an integer will be treated separately later.

Let $S_{1}$ be the characteristic root of largest real part, and let $\delta_{1}$ be the 
residue of $h^{\prime}(s)$ at $S_{1}$. We shall show later that $K\left(s, t_{1}\right)$ has a singularity at $S_{1}$ for every value of $t_{1}$, save possibly for a sequence of values of $t_{1}$ with sole limit point at infinity. Moreover, whether the singularity is a pole or a branch point depends only on $\delta_{1}$. For the time being, we assume this to be so. We remark that the possibility of the exceptional values of $t_{1}$ is of no importance in characterizing $k\left(t, t_{1}\right)$, since we have noted previously that $k$ is a continuous function.

We now define auxiliary functions as in $\$ 2$.

Case $1 . \operatorname{Re}\left(\delta_{1}\right)>-1, \delta_{1}$ not an integer. Let any positive integer $n$ be chosen such that $n+\operatorname{Re}\left(\delta_{1}\right)>1$, and let $\nu$ be the integer such that $\operatorname{Re}\left(\delta_{1}\right)-1$ $\leqq \nu<\operatorname{Re}\left(\delta_{1}\right)$. Define functions $r_{j}\left(t, S_{1}\right)(j=0,1, \cdots, \nu+n+2)$ as in equations (2.18) and (2.19), but with $S_{2}$ replaced by $S_{1}$ and $\delta_{2}$ by $\delta_{1}$. Define $r\left(t, S_{1}\right)$ by an equation analogous to (2.20), and let $R\left(s, S_{1}\right)$ be the transform of $r\left(t, S_{1}\right)$, and $R_{c}\left(s, S_{1}\right)$ the transform of $\bar{r}\left(t, S_{1}\right)$. We obtain an analogue of equation (2.21). Finally, define

$$
f\left(t, t_{1}\right)=\left\{\begin{array}{lr}
-J_{n}\left(t_{1}\right) r\left(t, S_{1}\right)-\bar{J}_{n}\left(t_{1}\right) \bar{r}\left(t, S_{1}\right) & \left(0 \leqq t<t_{0}\right) \\
-\frac{k\left(t, t_{1}\right)}{t_{1}}-J_{n}\left(t_{1}\right) r\left(t, S_{1}\right)-\bar{J}_{n}\left(t_{1}\right) \bar{r}\left(t, S_{1}\right) & \left(t_{1} \geqq t_{0}, t \geqq t_{0}\right)
\end{array}\right.
$$

and define $F\left(s, t_{1}\right)$ to be the transform of $f\left(t, t_{1}\right)$. We see that for $t_{1} \geqq t_{0}$

$$
F\left(s, t_{1}\right)=-\frac{K\left(s, t_{1}\right)}{t_{1}}-J_{n}\left(t_{1}\right) R\left(s, S_{1}\right)-\bar{J}_{n}\left(t_{1}\right) R_{c}\left(s, S_{1}\right) .
$$

Case 2. $\operatorname{Re}\left(\delta_{1}\right) \leqq-1, \delta_{1}$ not an integer. Our procedure is similar, and we omit the details.

The procedure of $\$ 2$ yields, in place of equation (2.25),

$$
f\left(t, t_{1}\right)=\frac{i^{n}}{2 \pi} \frac{e^{X_{1} t}}{t^{n}} \int_{-\infty}^{\infty} e^{i t y}\left\{\frac{d^{n}}{d y^{n}} F\left(X_{1}+i y, t_{1}\right)\right\} d y,
$$

where the boundary values of $F\left(s, t_{1}\right)$ are denoted by $F\left(X_{1}+i y, t_{1}\right)$. This formula holds for all $t_{1}>t_{0}$.

At this point, we must alter the procedure of $\$ 2$. Our next step is to prove the following lemma.

Lемма 4.1. If $\delta_{1}$ is not an integer, if $n$ is any positive integer for which $n+\operatorname{Re}\left(\delta_{1}\right)>1$, and if $t_{1}>t_{0}$, there are positive numbers $C$ and $C_{1}$, which do not depend on $t_{1}$ or $t$, such that $\left|J_{n}\left(S_{1}, t_{1}\right)\right| \leqq C t_{1}^{-\mathrm{Ro}\left(\delta_{1}\right)-C_{1}} e^{-X_{1} t_{1}}$.

$J_{n}\left(S_{1}, t_{1}\right)$ is defined by $(4.8)$ above. We suppose first that $\operatorname{Re}\left(\delta_{1}\right) \leqq 0$. If $N$ is the positive integer such that $N<n+\operatorname{Re}\left(\delta_{1}\right) \leqq N+1$, integration by parts yields 


$$
J_{n}\left(S_{1}, t_{1}\right)=t_{1}^{n-N-1} \int_{+\infty+i 0}^{S_{1}} H_{n-N-1}\left(s_{1}\right) e^{-s_{1} t_{1}} d s_{1} .
$$

Clearly the path may be deformed into the line from $+\infty+i Y_{1}$ to $S_{1}$. If $\operatorname{Re}\left(\delta_{1}\right)$ is not an integer, $\left|H_{n-N-1}\left(x_{1}+i Y_{1}\right)\right| \leqq C\left|x_{1}+i Y_{1}\right| c$, as may be deduced from (2.10) and the definition of $H_{j}(s)$, and the result follows at once. If $\operatorname{Re}\left(\delta_{1}\right)$ is an integer, (2.16) implies that $\left|H_{n-N-1}\left(x_{1}+i Y_{1}\right)\right| \leqq C$ for $x_{1}$ near $X_{1}$. If we split the integral into two parts, in one of which this bound may be employed, the stated result follows easily.

If $\operatorname{Re}\left(\delta_{1}\right)>0$, we have, instead,

$$
J_{n}\left(S_{1}, t_{1}\right)=t_{1}^{-(N-n+1)} \int_{+\infty+i 0}^{s_{1}} \frac{d^{N-n+1}}{d s_{1}^{N-n+1}}\left\{e^{h\left(s_{1}\right)}\right\} e^{-s_{1} t_{1}} d s_{1},
$$

since the first $N-n$ derivatives of $\exp \{h(s)\}$ approach zero as $s$ approaches $S_{1}$. We can now complete the proof just as before.

We shall also need the following result: If $\eta_{2}>Y_{1} \geqq 0$, there is a constant $C$, depending on $\eta_{2}$ but not on $\tau$, such that

$$
\begin{aligned}
& \mid \int_{-\infty}^{-\eta_{2}} \frac{e^{i \tau y}}{X_{1}+i y-b_{0}-a_{0} e^{-X_{1}-i y}} d y \\
& \quad+\int_{\eta_{2}}^{\infty} \frac{e^{i \tau y}}{X_{1}+i y-b_{0}-a_{0} e^{-X_{1}-i y}} d y \mid \leqq C
\end{aligned}
$$

for any $\tau>0$, or for any $\tau<0$. To prove this, it is clearly enough to consider

$$
I_{3}(\tau)=\int_{-\infty}^{-\eta_{2}} \frac{e^{i \tau y}}{y} d y+\int_{\eta_{2}}^{\infty} \frac{e^{i \tau y}}{y} d y .
$$

By integration by parts, we see that each of these integrals is uniformly convergent if $\tau$ is bounded away from zero, and approaches zero as $\tau \rightarrow \infty$. It will therefore suffice to show that $I_{3}(\tau)$ is bounded as $\tau \rightarrow+0$ and as $\tau \rightarrow-0$. Suppose that $\tau>0$, and consider the integral of $e^{z} / z$ over the contour in the $z$-plane which follows the imaginary axis from $-R i$ to $+R i$, except for an indentation on the semicircle $|z|=\tau \eta_{2}, \operatorname{Re}(z) \leqq 0$, then follows the horizontal straight segment from $R i$ to $-\sigma+R i$, then the vertical segment to $-\sigma-R i$, and finally the horizontal segment back to $-R i$. Since the value of this integral is zero, the stated result follows upon letting $R \rightarrow+\infty$ and then letting $\sigma \rightarrow+\infty$.

We shall now obtain a bound on $\left|f\left(t, t_{1}\right)\right|$, using (4.12). Define

$$
I_{4}(\alpha, \beta)=\int_{\alpha}^{\beta} e^{i t y}\left\{\frac{d^{n}}{d y^{n}} F\left(X_{1}+i y, t_{1}\right)\right\} d y .
$$

First we shall show that, given $\epsilon>0$, we may choose $\eta_{1}=\eta_{1}(\epsilon)$ and $\eta_{2}=\eta_{2}(\epsilon)$, 
with $0<\eta_{1}<Y_{1}<\eta_{2}$, such that $\left|I_{4}\left(\eta_{1}, \eta_{2}\right)\right|<\epsilon t_{1}^{n} e^{-X_{1} t_{1}}$ and $\left|I_{4}\left(-\eta_{2},-\eta_{1}\right)\right|$ $\left\langle\epsilon t_{1}^{n} e^{-X_{1} t_{1}}\right.$ for all $t>t_{1} \geqq t_{0}$. It will be enough to consider $I_{4}\left(\eta_{1}, \eta_{2}\right)$, and to restrict ourselves to the case in which $\operatorname{Re}\left(\delta_{1}\right)>-1$ and $\delta_{1}$ is not an integer. What we must prove is that for $y$ in some fixed interval about $Y_{1}$,

$$
\left|\frac{d^{n}}{d y^{n}} F\left(X_{1}+i y, t_{1}\right)\right| \leqq C t_{1}^{n} e^{-X_{1} t_{1}},
$$

where $C$ does not depend on $t_{0}, t, t_{1}$, or $y$. By (4.9) and (4.11),

(4.15) $F\left(s, t_{1}\right)=V_{n}\left(s, t_{1}\right)+Q_{n}\left(s, t_{1}\right)-\bar{J}_{n}\left(t_{1}\right) R_{c}\left(s, S_{1}\right)-J_{n}\left(t_{1}\right) \cdot$ entire function in the vicinity of $S_{1}$, where

$$
V_{n}\left(s, t_{1}\right)=\frac{e^{-h(s)}}{s-b_{0}-a_{0} e^{-s}}\left[e^{-s t_{1}} \sum_{j=1}^{n} H_{j}(s) t_{1}^{j-1}+t_{1}^{n} \int_{S_{1}}^{s} H_{n}\left(s_{1}\right) e^{-s_{1} t_{1}} d s_{1}\right],
$$

and

$$
Q_{n}\left(s, t_{1}\right)=J_{n}\left(t_{1}\right)\left(s-S_{1}\right)^{-\delta_{1}-1} \sum_{j=\nu+n+3}^{\infty} h_{j}\left(s-S_{1}\right)^{j} .
$$

Since $n+\operatorname{Re}\left(\delta_{1}\right)>1$, and since $R_{c}\left(s, S_{1}\right)$ is analytic near $S_{1}$, Lemma 4.1 shows that the contribution of the last two terms in (4.15) satisfies (4.14). The term arising from $Q$ is bounded as required by choice of $\nu$. Finally, $V_{n}$ may be handled by a calculation using the series representations (2.8), (2.9), and (2.16).

Next we shall prove that $\left|I_{4}\left(-\eta_{1}, \eta_{1}\right)\right| \leqq C t_{1}^{n-1} e^{-X_{1} t_{1}}$ if $0<\eta_{1}<Y_{1}$, where $C$ depends on $\eta_{1}$ but not on $t, t_{1}$, or $t_{0}$. It will be enough to show that the derivative in the integrand is so bounded for $|y| \leqq \eta_{1}$. Since the boundary functions of $K, R$, and $R_{c}$ exist separately over this range, we see from Lemma 4.1 that we need only consider the $n$th derivative of $-K\left(X_{1}+i y, t_{1}\right) / t_{1}$. From equation (4.7) we obtain

$$
\begin{aligned}
& \frac{d^{n}}{d s^{n}}\left\{-\frac{K\left(s, t_{1}\right)}{t_{1}}\right\}=\frac{d^{n}}{d s^{n}}\left(\frac{e^{-h}}{\zeta}\right) \int_{L_{2}} e^{h\left(s_{1}\right)} e^{-s_{1} t_{1}} d s_{1} \\
&+e^{-8 t_{1}} \sum_{\mu=1}^{n}\left(\begin{array}{c}
n \\
\mu
\end{array}\right) \frac{d^{n-\mu}}{d s^{n-\mu}}\left(\frac{e^{-h}}{\zeta}\right) \sum_{\nu=0}^{\mu-1}\left(-t_{1}\right)^{\nu}\left(\begin{array}{c}
\mu-1 \\
\nu
\end{array}\right) \frac{d^{\mu-1-\nu}}{d s^{\mu-1-\nu}}\left(e^{h}\right)
\end{aligned}
$$

where $\zeta(s)=s-b_{0}-a_{0} e^{-8}$. Since all the functions of $s$ in (4.16) are bounded for $s=X_{1}+i y,|y| \leqq \eta_{1}$, the desired result is clear.

Finally we shall prove that if $\eta_{2}>Y_{1} \geqq 0$, there is a constant $C$, depending on $\eta_{2}$ but not on $t, t_{1}$; or $t_{0}$, such that

$$
\left|I_{4}\left(-\infty,-\eta_{2}\right)+I_{4}\left(\eta_{2}, \infty\right)\right| \leqq C t_{1}^{n-1} e^{-X_{1} t_{1}}
$$

for $t>t_{1} \geqq t_{0}$. To do this, we first consider 


$$
I_{5}=\int_{-\infty}^{-\eta_{2}} e^{i t y}\left\{\frac{d^{n}}{d y^{n}} \frac{K\left(X_{1}+i y, t_{1}\right)}{-t_{1}}\right\} d y+\int_{\eta_{2}}^{\infty} e^{i t y}\left\{\frac{d^{n}}{d y^{n}} \frac{K\left(X_{1}+i y, t_{1}\right)}{-t_{1}}\right\} d y .
$$

The $n$th derivative of $K\left(s, t_{1}\right) / t_{1}$ is given by equation (4.16). The portion of $I_{5}$ due to the term for which $\mu=n, \nu=n-1$, has the desired bound by virtue of (4.13). For the other terms, we proceed in a manner analogous to that used in $\$ 2$ to prove that $U\left(X_{2}+i y\right)$ and its first $n-1$ derivatives approach zero as $|y| \rightarrow \infty$. The proof of (4.17) may then be completed with the aid of Lemma 4.1.

By combining the above results, we find that, given $\epsilon>0$,

$$
\left|I_{4}(-\infty, \infty)\right| \leqq C(\epsilon) t_{1}^{n-1} e^{-X_{1} t_{1}}+\epsilon t_{1}^{n} e^{-X_{1} t_{1}}, \text { for } t>t_{1} \geqq t_{0},
$$

where $C(\epsilon)$ does not depend on $t_{0}, t$, or $t_{1}$. This inequality, in combination with (4.12), provides a useful bound on $f\left(t, t_{1}\right)$. By proceeding somewhat as in $\S 2$, we can obtain a similar result in case $\delta_{1}$ is an integer. We omit the details. The final conclusions are summarized in the theorem which follows.

TheOREM 4.1. Let $S_{1}$ be the characteristic root of largest real part and let $\delta=\delta_{1}$ be the residue of $h^{\prime}(s)$ at $S_{1}$.

(1) If $\delta$ is a non-negative integer,

$$
-\frac{k\left(t, t_{1}\right)}{t_{1}}=J\left(t_{1}\right) r\left(t, S_{1}\right)+f\left(t, t_{1}\right)
$$

where

$$
\begin{aligned}
J\left(t_{1}\right)= & \int_{+\infty+i 0}^{S_{1}} e^{h\left(s_{1}\right)} e^{-s_{1} t_{1}} d s_{1}, \\
r\left(t, S_{1}\right)= & t^{\delta} e^{S_{1} t}\left(C_{0}+\frac{C_{1}}{t}+\cdots+\frac{C_{\delta}}{t^{\delta}}\right), \\
& \left|f\left(t, t_{1}\right)\right| \leqq C e^{x\left(t-t_{1}\right)}
\end{aligned}
$$$$
\left(x<X_{1}\right) .
$$

(2) If $\delta$ is a negative integer,

$$
-\frac{k\left(t, t_{1}\right)}{t_{1}}=J\left(t_{1}\right) r\left(t, S_{1}\right)+\bar{J}\left(t_{1}\right) \bar{r}\left(t, S_{1}\right)+f\left(t, t_{1}\right)
$$

where

$$
r\left(t, S_{1}\right)=t^{\delta} e^{S_{1} t}\left(C_{0}+\frac{C_{1}}{t}+\cdots+\frac{C_{\delta+n-1}}{t^{\delta+n-1}}\right)
$$

and where 


$$
J\left(t_{1}\right)=\frac{1}{2 \pi i} \int e^{h(s)} e^{-8 t_{1}} d s=t^{-\delta-1} e^{-S_{1} t_{1}}\left(C_{0}+\cdots+\frac{C_{-\delta-1}}{t_{1}^{-\delta-1}}\right),
$$

the integral being taken around a small circle surrounding $S_{1}$. Also, given $\epsilon>0$, there exists $C(\boldsymbol{\epsilon})$ such that

$$
\left|f\left(t, t_{1}\right)\right| \leqq \frac{t_{1}^{n-1}}{t^{n}} e^{X_{1}\left(t-t_{1}\right)}\left[C(\epsilon)+\epsilon t_{1}\right]
$$

for $t_{1}>t \geqq t_{0}$ and any positive integer $n$ for which $n+\operatorname{Re}\left(\delta_{1}\right)>1$.

(3) If $\delta$ is not an integer, the results are as in (2), except that $J\left(t_{1}\right)=J_{n}\left(S_{1}, t_{1}\right)$ is defined by (4.8) and

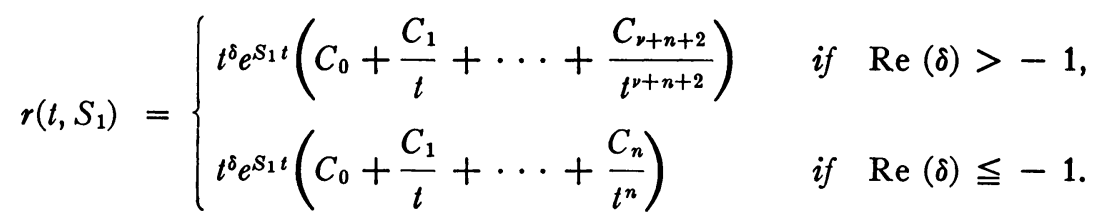

The above theorem was derived under the assumption that $K\left(s, t_{1}\right)$ has a singularity at $S_{1}$ for every value of $t_{1}$, save possibly for a sequence of values of $t_{1}$ with sole limit point at infinity. We shall now prove that this is true. We first observe from the above discussion that a necessary and sufficient condition in order that $K\left(s, t_{1}\right)$ should have a singularity at $S_{1}$ and $\bar{S}_{1}$, for a fixed $t_{1}$, is that $J\left(t_{1}\right) \neq 0$. Let us now consider the function

$$
I_{6}\left(S_{1}, t_{1}\right)=\int_{+\infty+i 0}^{S_{1}} H_{n}(\sigma) e^{-\sigma t_{1}} d \sigma
$$

which, for the moment, we regard as a function of the complex variable $t_{1}$. Since the integral is uniformly convergent for $\operatorname{Re}\left(t_{1}\right)$ bounded away from zero, $I_{6}$ is an analytic function of $t_{1}$ for $\operatorname{Re}\left(t_{1}\right)>0$. Consequently $I_{6}$, as a function of the real variable $t_{1}$, can be zero only for a sequence of values of $t_{1}>0$ with sole limit point at infinity, unless it is identically zero. If $\delta_{1}$ is an integer, the same argument may be applied to the corresponding integral in the definition of $J$. Thus in order to complete the proof we need only show that the appropriate integral cannot be zero for all $t_{1}>0$. Let us suppose that $\delta_{1}$ is not an integer and that $I_{6}\left(S_{1}, t_{1}\right)=0$ for all $t_{1}>0$. Then, regarding $t_{1}$ as a complex variable,

$$
-e^{i Y_{1} t_{1}} I_{6}=\int_{X}^{\infty} H_{n}\left(x+i Y_{1}\right) e^{-x t_{1}} d x
$$

is zero for $\operatorname{Re}\left(t_{1}\right)>0$. Let 


$$
\Phi\left(t_{1}\right)=\int_{0}^{\infty} \phi(x) e^{-x t_{1}} d x
$$

where

$$
\phi(x)=\left\{\begin{array}{cl}
\operatorname{Re}\left\{H_{n}\left(x+i Y_{i}\right)\right\} & \text { if } \quad x>X_{1}, \\
0 & \text { if } \quad 0<x<X_{1} .
\end{array}\right.
$$

If $I_{6}$ is identically zero, then $\Phi\left(t_{1}\right)$ is zero for $\operatorname{Re}\left(t_{1}\right)>0$. But $\Phi\left(t_{1}\right)$ is the transform of $\phi(x)$. It follows from Doetsch [5, p. 135, Theorem 2], that $\phi(x)$ is identically zero. This is a contradiction. Hence $I_{6}$ is not identically zero. A similar argument may be used if $\delta_{1}$ is an integer. The proof of Theorem 4.1 is therefore complete.

We can now prove Theorem 1.2. We shall construct a solution of the integral equation (4.5), using the classical method of successive approximations. Define

$$
\begin{aligned}
u_{m+1}(t)= & u_{0}(t)=g(t) \quad\left(t_{0} \leqq t \leqq t_{0}+1 ; m=0,1,2, \cdots\right), \\
u_{m+1}(t+1)= & u_{0}(t+1) \\
& +\int_{t_{0}}^{t}\left\{A\left(t_{1}\right) u_{m}\left(t_{1}\right)+B\left(t_{1}\right) u_{m}\left(t_{1}+1\right)\right\} k\left(t, t_{1}\right) d t_{1} \\
& \left(t>t_{0} ; m=0,1,2, \cdots\right) .
\end{aligned}
$$

$u_{0}(t)$ is the solution of the equation of first approximation. Note that the boundary condition (4.3) is satisfied by each iterate.

According to the results of $\$ 2$,

$$
\left|u_{0}(t+1)\right| \leqq C t^{\operatorname{Re}\left(\delta_{2}\right)} e^{X_{2} t} \leqq \alpha_{1} t^{\operatorname{Re}\left(\delta_{1}\right)} e^{X_{1} t}
$$

for $t \geqq t_{0}-1$. We shall now prove by induction that for $t>t_{0}$

$$
\left|u_{m}(t+1)\right| \leqq 5 \alpha_{1} t^{\mathrm{Re}\left(\delta_{1}\right)} e^{X_{1} t},
$$

from which it will follow, since $u_{m}(t)=u_{0}(t)$ for $t_{0} \leqq t \leqq t_{0}+1$, that there is a constant $\alpha_{2}$ such that

$$
\left|u_{m}(t)\right| \leqq 5 \alpha_{1} \alpha_{2} t^{\mathrm{Re}\left(\delta_{1}\right)} e^{X_{1} t}
$$

for $t \geqq t_{0}$. As just observed, (4.29) is true for $m=0$, and hence so is (4.30). We suppose that they have been proved for $m=0,1, \cdots, p$, and attempt to prove (4.29) for $m=p+1$. By substituting for $k\left(t, t_{1}\right)$ in (4.27), using (4.18) or (4.22), and by observing from (4.4) that

$$
|A(t)| \leqq \frac{A}{t^{2}}, \quad|B(t)| \leqq \frac{B}{t^{2}},
$$

we obtain 


$$
\begin{aligned}
\left|u_{p+1}(t+1)\right| \leqq & \left|u_{0}(t+1)\right|+2 A\left|r\left(t, S_{1}\right)\right| \int_{t_{0}}^{t} t_{1}^{-1}\left|J\left(t_{1}\right)\right|\left|u_{p}\left(t_{1}\right)\right| d t_{1} \\
& +2 B\left|r\left(t, S_{1}\right)\right| \int_{t_{0}}^{t} t_{1}^{-1}\left|J\left(t_{1}\right)\right|\left|u_{p}\left(t_{1}+1\right)\right| d t_{1} \\
& +A \int_{t_{0}}^{t} t_{1}^{-1}\left|f\left(t, t_{1}\right)\right|\left|u_{p}\left(t_{1}\right)\right| d t_{1} \\
& +B \int_{t_{0}}^{t} t_{1}^{-1}\left|f\left(t, t_{1}\right)\right|\left|u_{p}\left(t_{1}+1\right)\right| d t_{1} .
\end{aligned}
$$

Each term in (4.32) may be shown to be bounded by $\alpha_{1} t^{\operatorname{Re}\left(\delta_{1}\right)} e^{X_{1} t}$ by using the following inequalities:

$$
\begin{aligned}
\left|r\left(t, S_{1}\right)\right| & \leqq C t^{\operatorname{Re}\left(\delta_{1}\right)} e^{X_{1} t} & (t>1), \\
\left|J\left(t_{1}\right)\right| & \leqq C t_{1}^{-C-\operatorname{Re}\left(\delta_{1}\right)} e^{-X_{1} t_{1}} & \left(t_{1}>t_{0}\right) .
\end{aligned}
$$

The first comes from Theorem 4.1 and the second from Lemma 4.1 and Theorem 4.1. In treating the last two integrals in (4.32) when $\delta_{1}$ is not an integer, or when $\delta_{1}$ is a negative integer, we use the bound (4.25), with $\epsilon=1 / 10 A \alpha_{2}$, and the fact that $n+\operatorname{Re}\left(\delta_{1}\right)>1$. Thus (4.29) and (4.30) are proved.

The successive approximations defined by (4.27) obviously converge to $g(t)$ for $t_{0} \leqq t \leqq t_{0}+1$. We shall now prove that they converge for $t>t_{0}+1$ by proving that the series

$$
\sum_{m=0}^{\infty}\left\{u_{m+1}(t+1)-u_{m}(t+1)\right\}
$$

converges for $t>t_{0}$. We can prove by induction, in much the same way (4.29) was established, that

$$
\left|u_{m+1}(t+1)-u_{m}(t+1)\right| t^{-\operatorname{Re}\left(\delta_{1}\right)} e^{-X_{1} t} \leqq 10 \frac{\alpha_{3}^{m}\left(t-t_{0}\right)^{m}}{m !}
$$

for $t>t_{0}, m=0,1,2, \cdots \alpha_{3}$ is a constant which does not depend on $m$. (4.36) shows immediately that (4.35) is uniformly convergent in any finite interval in which $t>t_{0}$. Thus the sequence $u_{0}(t+1), u_{1}(t+1), \cdots$, of approximations converges uniformly in every finite interval to a certain function $u(t+1)$.

It is clear that $u(t+1)$ is continuous for $t>t_{0}$, and, by (4.30), that

$$
|u(t)| \leqq C t^{\mathrm{Re}\left(\delta_{1}\right)} e^{X_{1} t}
$$

By reason of the definitions (4.27) and the uniformity of convergence, $u(t)$ satisfies the boundary condition (4.3) and for $t>t_{0}$ it satisfies the integral equation (4.5). Finally, because of (4.37), the integral 


$$
\int_{t_{0}}^{\infty} t_{1}\left\{A\left(t_{1}\right) u\left(t_{1}\right)+B\left(t_{1}\right) u\left(t_{1}+1\right)\right\} e^{-s t_{1}} d t_{1}
$$

is absolutely convergent for $\operatorname{Re}(s)>X_{1}$. We can therefore prove that $u(t)$ satisfies the differential-difference equation (4.1) for $t>t_{0}$ by retracing the discussion in \$3. This completes the proof of Theorem 1.2.

5. The nonlinear equation. We shall now discuss the nonlinear equation

$$
\frac{d}{d t} u(t+1)=a(t) u(t)+b(t) u(t+1)+D(u(t), u(t+1))
$$

under the conditions stated in Theorem 1.3. We wish to examine the behavior, as $t \rightarrow+\infty$, of a function $u(t)$ which satisfies (5.1) for $t>t_{0}$ and which satisfies

$$
u(t)=g(t)
$$

for $t_{0} \leqq t \leqq t_{0}+1$. Since (5.1) may be written in the form of equation (3.1), with $w(t)=A(t) u(t)+B(t) u(t+1)+D(u(t), u(t+1))$ and

$$
A(t) \sim \frac{a_{2}}{t^{2}}+\frac{a_{3}}{t^{3}}+\cdots, \quad B(t) \sim \frac{b_{2}}{t^{2}}+\frac{b_{3}}{t^{3}}+\cdots,
$$

as $t \rightarrow+\infty$, the results of $\S 3$ suggest the consideration of the integral equation

$$
\begin{aligned}
u(t+1)=u_{0}(t+1)+\int_{t_{0}}^{t}\left\{A\left(t_{1}\right) u\left(t_{1}\right)\right. & +B\left(t_{1}\right) u\left(t_{1}+1\right) \\
& \left.+D\left(u\left(t_{1}\right), u\left(t_{1}+1\right)\right)\right\} k\left(t, t_{1}\right) d t_{1} .
\end{aligned}
$$

We shall now construct a solution of (5.4) by the method of successive approximations. Define

$$
\begin{aligned}
u_{m+1}(t)= & u_{0}(t)=g(t) \quad\left(t_{0} \leqq t \leqq t_{0}+1 ; m=0,1,2, \cdots\right), \\
u_{m+1}(t+1)= & u_{0}(t+1)+\int_{t_{0}}^{t}\left\{A\left(t_{1}\right) u_{m}\left(t_{1}\right)+B\left(t_{1}\right) u_{m}\left(t_{1}+1\right)\right. \\
& \left.+D\left(u_{m}\left(t_{1}\right), u_{m}\left(t_{1}+1\right)\right)\right\} k\left(t, t_{1}\right) d t_{1} \\
& \left(t>t_{0} ; m=0,1,2, \cdots\right) .
\end{aligned}
$$

As in $\S 4$, our first aim is to establish a uniform bound on the iterates. We shall prove by induction that

$$
\left|u_{m}(t+1)\right| \leqq 7 \alpha_{1} t^{\mathrm{Re}\left(\delta_{1}\right)} e^{X_{1} t}
$$

for $t>t_{0}, m=0,1,2, \cdots$, and that

$$
\left|u_{m}(t)\right| \leqq 7 \alpha_{1} \alpha_{2} t^{R \theta\left(\delta_{1}\right)} e^{X_{1} t}
$$

for $t \geqq t_{0}, m=0,1,2, \cdots \alpha_{1}$ and $\alpha_{2}$ are the constants in equations (4.29) 
and (4.30). The proof is just like the corresponding proof in $\$ 4$ except that we must establish a suitable bound on $\left|D_{p}\right|=\left|D\left(u_{p}(t), u_{p}(t+1)\right)\right| .(5.6)$ and (5.7) certainly hold for $m=0$. Assume that they hold for $m=0,1, \cdots, p$. Then, from the definition of $D$,

$$
\left|D_{p}\right| \leqq \sum_{i+j \geqq 2} b_{i j}\left(7 \alpha_{1} \alpha_{2} t^{\mathrm{Re}\left(\delta_{1}\right)} e^{X_{1} t}\right)^{i}\left(7 \alpha_{1} t^{\mathrm{Re}\left(\delta_{1}\right)} e^{X_{1} t}\right)^{j}
$$

We wish, first of all, to show that this series converges for $t \geqq t_{0}$, provided

$$
\max _{t_{0} \leqq t \leqq t_{0}+1}|g(t)|
$$

is sufficiently small. Now from equation (2.5) and the definition of $J\left(S_{2}, t_{0}\right)$ we see that $\left|J\left(S_{2}, t_{0}\right)\right| \leqq C$, where $C$ can be made as small as desired by taking $\max |g(t)|$ sufficiently small. Furthermore, by re-examining $\$ 2$, we see that

$$
\left|f\left(t-1, t_{0}\right)\right|=t^{-n} e^{-X_{2} t} \epsilon(t),
$$

where the maximum of $|\epsilon(t)|$ for $t \geqq t_{0}$ is small if $\max |g(t)|$ is small. It follows that in equation (4.29) $\alpha_{1}$ is as small as desired if $\max |g(t)|$ is sufficiently small.

It follows that the series (5.8) is convergent for all $t \geqq t_{0}$ if $\max |g(t)|$ is small enough. In fact,

$$
\left|D_{p}\right| \leqq \alpha_{1} \alpha_{5}\left(t^{\mathrm{Re}\left(\delta_{1}\right)} e^{X_{1} t}\right)^{3 / 2}
$$

for $t \geqq t_{0}$, where $\alpha_{5}$ is small if $\max |g(t)|$ is small. Using (5.9) and the fact that $\alpha_{5}$ may be taken as small as is required, we can complete the inductive proof of (5.6) and (5.7) just as in \$4.

We show next that (5.5) defines a convergent sequence. The method is the same as that used in the preceding section. We shall first prove by induction that for $t>t_{0}, m=0,1,2, \cdots$,

$$
\left|u_{m+1}(t+1)-u_{m}(t+1)\right| t^{-\mathrm{Re}\left(\delta_{1}\right)} e^{-X_{1} t} \leqq \frac{14 \alpha_{1} \alpha_{6}^{m} t^{2 m}}{m !}
$$

for a certain constant $\alpha_{6}$. (5.10) is certainly true for $m=0$. Suppose that it has been proved for $m=0,1,2, \cdots, p-1$. We consider the quantity

$$
\left|D_{p}-D_{p-1}\right|=\left|D\left(u_{p}(t), u_{p}(t+1)\right)-D\left(u_{p-1}(t), u_{p-1}(t+1)\right)\right| \text {. }
$$

Note that because of the uniform bound on the approximations just established, the series $D_{p}$ and $D_{p-1}$ converge absolutely for $t \geqq t_{0}$, provided $\max |g(t)|$ is sufficiently small. Now by the mean value theorem and the inequalities (5.6) and (5.7), we see that

$$
\left|D_{p}-D_{p-1}\right| \leqq C t^{\mathrm{Re}\left(\delta_{1}\right)} e^{X_{1} t}\left\{\left|u_{p}(t)-u_{p-1}(t)\right|+\left|u_{p}(t+1)-u_{p-1}(t+1)\right|\right\} \text {. }
$$

By the assumption that (5.10) holds for $m=p-1$, 


$$
\left|D_{p}-D_{p-1}\right| \leqq \frac{14 C \alpha_{1} \alpha_{6}^{p-1}}{(p-1) !} t^{\operatorname{Re}\left(\delta_{1}\right)+2 p-2} e^{X_{1} t}
$$

The induction may now be completed as before.

Just as in $\$ 4$, the inequality (5.10) shows that the sequence of approximations $u_{0}(t+1), u_{1}(t+1), \cdots$ converges to a limit function $u(t+1)$ for $t>t_{0}$, and the convergence is uniform in any finite interval in which $t>t_{0} . u(t+1)$ is continuous for $t>t_{0}$, satisfies (5.4) for $t>t_{0}$, satisfies (5.2), and

$$
|u(t+1)| \leqq 7 \alpha_{1} t^{\operatorname{Re}\left(\delta_{1}\right)} e^{X_{1} t} .
$$

Finally, (5.11) enables us to prove, by the method used in proving (5.9), that

$$
|D(u(t), u(t+1))| \leqq C\left(t^{\mathrm{Re}\left(\delta_{1}\right)} e^{X_{1} t}\right)^{3 / 2} .
$$

Consequently the integral

$$
\int_{t_{0}}^{\infty} t_{1}\left\{A\left(t_{1}\right) u\left(t_{1}\right)+B\left(t_{1}\right) u\left(t_{1}+1\right)+D\left(u\left(t_{1}\right), u\left(t_{1}+1\right)\right)\right\} e^{-s t_{1}} d t_{1}
$$

is absolutely convergent for $\operatorname{Re}(s)>X_{1}$. We can therefore prove that $u(t)$ satisfies equation (5.1) for $t>t_{0}$ by retracing the steps of $\S 3$.

Next we shall prove that the function $u(t)$ constructed above by successive approximations is the unique solution of (5.1) and (5.2). Suppose that $v(t)$ is another solution, and that $v(t)=u(t)$ for $t_{0} \leqq t \leqq T_{0}\left(T_{0} \geqq t_{0}+1\right)$, but that $v$ and $u$ are not equal in any larger interval. From (5.11), $|u(t)| \leqq C_{1}$ for $t_{0} \leqq t$ $\leqq T_{0}+1$, where $C_{1}$ is as small as desired if $\max |g(t)|$ is sufficiently small. Since $u(t)$ and $v(t)$ are continuous, there is an $\epsilon, 0<\epsilon<1$, such that $|v(t)| \leqq 2 C_{1}$ for $t_{0} \leqq t \leqq T_{0}+\epsilon$. Hence $D(u(t), u(t+1))$ and $D(v(t), v(t+1))$ are absolutely convergent for $t_{0} \leqq t \leqq T_{0}-1+\epsilon$, and $|D(u(t), u(t+1))-D(v(t), v(t+1))|$ $\leqq C_{2}|u(t+1)-v(t+1)|$ for $t_{0} \leqq t \leqq T_{0}-1+\epsilon$. Now from equation (5.1) we see that $u$ and $v$ are both solutions of the integral equation

$$
\begin{aligned}
u(t+1)=g\left(t_{0}+1\right)+\int_{t_{0}}^{t}\left\{a\left(t_{1}\right) u\left(t_{1}\right)+b\left(t_{1}\right) u\left(t_{1}\right.\right. & +1) \\
& \left.+D\left(u\left(t_{1}\right), u\left(t_{1}+1\right)\right)\right\} d t_{1} .
\end{aligned}
$$

Consequently

$$
|u(t+1)-v(t+1)| \leqq C_{3} \int_{T_{0}-1}^{t}\left|u\left(t_{1}+1\right)-v\left(t_{1}+1\right)\right| d t_{1}
$$

for $T_{0}-1 \leqq t \leqq T_{0}-1+\epsilon$. But $\left|u\left(t_{1}\right)-v\left(t_{1}\right)\right| \leqq 4 C_{1}$ for $T_{0} \leqq t_{1} \leqq T_{0}+\epsilon$. It follows from iteration in (5.12) that $u(t)=v(t)$ for $T_{0} \leqq t \leqq T_{0}+\epsilon$. This contradicts our original assumption. Therefore the solution $u(t)$ is unique.

The proof of Theorem 1.3 is now complete. 


\section{REFERENCES}

1. R. Bellman, The boundedness of solutions of linear differential equations, Duke Math. J. vol. 14 (1947) pp. 83-97.

2. - On the existence and boundedness of solutions of non-lirear differential-difference equations, Ann. of Math. vol. 50 (1949) pp. 347-355.

3. S. Bochner, Allgemeine lineare Differenzgleichungen mit asymptotisch konstanten Koeffzienten, Math. Zeit. vol. 33 (1931) pp. 426-450.

4. F. H. Brownell, Non-linear delay differential equations, "Contributions to the Theory of Non-linear Oscillatiơns," Annals of Mathematics Studies, no. 20, Princeton, 1950, pp. 89-148.

5. G. Doetsch, Theorie und Anwendung der Laplace-Transformation, New York, 1943.

6. N. D. Hayes, Roots of the transcendental equation associated with a certain differencedifferential equation, J. London Math. Soc. vol. 25 (1950) pp. 226-232.

7. O. Hilb, Zur Theorie der linearen funktionale Differentialgleichungen, Math. Ann. vol. 78 (1917) pp. 137-170.

8. G. Hoheisel, Randwertaufgaben und funktionale Differentialgleichungen, Jber. Deutschen. Math. Verein. vol. 39 (1930) pp. 54-58.

9. M. Hukuhara, Sur les points singuliers des equations differentielles lineaires, Journal of the Faculty of Science. Hokaido Imperial University Ser. I, vol. 2 (1934) pp. 13-88.

10. H. R. Pitt, On a class of linear integro-differential equations, Proc. Cambridge Philos. Soc. vol. 40 (1944) pp. 199-211.

11. E. Schmidt, Über eine Klasse linearer funktionaler Differentialgleichungen, Math. Ann. vol. 70 (1911) pp. 499-524.

12. E. C. Titchmarsh, Solutions of some functional equations, J. London Math. Soc. vol. 14 (1939) pp. 118-124.

13. W. J. Trjitzinsky, Analytic theory of linear differential equations, Acta Math. vol. 62 (1934) pp. 167-226.

14. E. M. Wright, The non-linear difference-differential equation, Quart. J. Math. Oxford Ser. (1) vol. 17 (1946) pp. 245-252.

15. - Linear difference-differential equations, Proc. Cambridge Philos Soc. vol. 44 (1948) pp. 179-185.

16. - The linear difference-differential equation with asymptotically constant coefficients, Amer. J. Math. vol. 70 (1948) pp. 221-238.

17. - The linear difference-differential equation with constant coefficients, Proceedings of the Royal Society of Edinburgh, Sec. A vol. 62 (1949) pp. 387-393.

18. - The stability of solutions of non-linear difference-differential equations, Proceedings of the Royal Society of Edinburgh, Sec. A vol. 63 (1950) pp. 18-26.

STANFORd UNIVERSITY, Stanford, Calif. 